\title{
Recalibrating Valence Weighting Tendencies as a Means of Reducing Anticipated Discomfort with an Interracial Interaction
}

\author{
${ }^{1}$ Evava S. Pietri \\ ${ }^{2}$ John F. Dovidio \\ ${ }^{3}$ Russell H. Fazio \\ ${ }^{1}$ Indiana University Purdue University Indianapolis \\ ${ }^{2}$ Yale University \\ ${ }^{3}$ The Ohio State University
}

\begin{abstract}
Author Note
We would like to thank the Yale Intergroup Relations lab for their valuable input on this research. This research was supported in part by Alfred P. Sloan Foundation grant \#213-3-15 awarded to the second author, and Howard Hughes Medical Institute. The authors have no conflict of interest to report.

Please address correspondence to Evava S. Pietri, Indiana University Purdue University Indianapolis, Department of Psychology, LD 124, 402 N. Blackford Street, Indianapolis, IN 46202, email:epietri@iupui.edu, phone:317-274-6753
\end{abstract}

Key words: attitude generalization; interracial interaction; valence weighting

Word count: Abstract=148, Main text=7,197, Footnotes=239, References=1,202, Tables=114 Table and Figures: 1 Table, 5 Figures

This is the author's manuscript of the article published in final edited form as:

Pietri, E. S., Dovidio, J. F., \& Fazio, R. H. (2018). Recalibrating valence-weighting tendencies as a means of reducing anticipated discomfort with an interracial interaction. Group Processes \& Intergroup Relations, 21(4), 597-614. https://doi.org/10.1177/1368430216674341 
Recalibrating Valence Weighting and Interracial Interaction

\begin{abstract}
We utilized a general intervention that affects (through “recalibration”) the way people generalize negative associations when evaluating objects to promote less negative expectations about an interaction with a Black internet "chat” partner. During this intervention, participants played a game to learn which "beans" varying in shape and speckles increased or decreased their points. Participants later classified game and new beans as good or bad. Recalibration condition participants were told whether they classified beans correctly, thus receiving feedback regarding the appropriate weighting of resemblance to a known positive versus a negative. Control participants, who received no feedback, were more likely to classify new beans as negative than recalibration participants. Compared to control, the recalibration condition also anticipated feeling less intergroup anxiety during a chat with a Black partner (Experiments 1 and 2) and this effect was strongest among participants who reported fewer close interactions with Black people (Experiment 2).
\end{abstract}


Recalibrating Valence Weighting and Interracial Interaction

\section{Recalibrating Valence Weighting Tendencies as a Means of Reducing Anticipated Discomfort with an Interracial Interaction}

Positive contact between members of different groups, predominantly under the conditions (e.g., equal status) specified by contact theory, is one of the most effective ways to improve intergroup attitudes (Dovidio, Gaertner, \& Kawakami, 2003; Pettigrew \& Tropp, 2011; Shook, \& Fazio, 2008). Even an online interaction can have positive consequences for intergroup attitudes (Amichai-Hamburger \& McKenna, 2006). However, both structural (e.g., racial segregation; Rugh \& Massey, 2014) and psychological factors (e.g., intergroup anxiety) limit such interactions. To help address the latter obstacle, the present research investigated how modifying individuals' generalization of positive and negative associations can influence the willingness of Whites to engage in an interaction with a Black individual.

Negative beliefs about intergroup contact often prevent individuals from participating in these exchanges. For example, when thinking about interacting with a Black individual, Whites often report fear of rejection (Shelton, 2003; Shelton \& Richeson, 2005; Vorauer \& Kumhyr, 2001), anxiety about how effectively and comfortably they can interact with Blacks (Gaertner \& Dovidio, 1986; Migacheva \& Tropp, 2012; Towles-Schwen \& Fazio, 2003), and apprehension that they will have nothing in common (Mallett, Wilson, \& Gilbert, 2008). Although there are also benefits associated with intergroup contact (i.e., learning about members from another group; Migacheva \& Tropp, 2012), Whites tend to let the bad aspects of intergroup intergroup interactions outweigh the good (Shelton \& Richeson, 2005). For example, Whites assume they will experience more negative emotions during an encounter with a Black than White individual (Mallett et al., 2008). 
Recalibrating Valence Weighting and Interracial Interaction

This anticipated emotion, often in the form of anxiety, ultimately decreases the desire to interact with a Black person in the future (Plant, 2004; Plant, Butz, \& Tartakovsky, 2008; Plant \& Devine, 2003) and results in fewer friendships with members from different groups (Mallett \& Wilson, 2010). Negative expectations can, thus, have broad intergroup consequences because forming a friendship with a member of another group is one of the most potent factors for developing more positive attitudes toward the group as a whole (Pettigrew, 1998; Paolini, Hewstone, Cairns, \& Voci, 2004; Turner, Hewstone, Voci, Paolini, \& Christ, 2007). Understanding the processes influencing these adverse expectancies is, therefore, critical for promoting contact and positive attitudes between groups.

Similar to an assessment of any novel occurrence, evaluating a potential interaction with an outgroup member represents an exercise in attitude generalization (Fazio, Pietri, Rocklage, \& Shook, 2015). Does the novel situation more closely resemble past occurrences associated with a positive attitude or a negative attitude? Although differences in valence weighting play a role in such assessments (for a review, see Fazio et al., 2015), negative associations typically generalize more strongly than positive associations (Fazio, Eiser, \& Shook, 2004; Shook, Fazio, \& Eiser, 2007; Pietri, Fazio, \& Shook, 2013b). This tendency in attitude generalization suggests Whites may generalize their past discomfort in intergroup encounters more strongly than their previous enjoyable encounters when predicting how they will feel during a new interaction with a Black person (Mallett et al., 2008). If this possibility is true, then encouraging less negative attitude generalization tendencies could reduce anxiety.

A recalibration exercise was developed specifically to alter the overgeneralization of negative associations with novel objects. This exercise involves playing a computer game, affectionately called BeanFest (see Fazio, Shook, \& Eiser, 2004), during which participants 
Recalibrating Valence Weighting and Interracial Interaction

approach or avoid “beans,” varying on a 10 (circular to oblong) x 10 (few to many speckles)

matrix in order to gain points and avoid losing points. Following the game, participants classify

as good and bad (i.e., ones that would increase or decrease points) the game beans and new beans from the matrix that look similar to both the positive and negative game beans (see Figure 1 for example of beans).

As previously mentioned, participants tend to generalize their negative attitudes more strongly and classify more novel beans as negative (Fazio et al., 2015; Pietri, Fazio, \& Shook, 2013b). However, it is possible to recalibrate individuals to reduce this negativity bias by informing individuals whether they correctly or incorrectly identify the valence of the game beans and the new beans (based on their proximity to the game beans in the matrix). By receiving feedback, which helps individuals correct their inaccuracies due to the negativity bias, participants begin to generalize their negative and positive attitudes more equivalently and are no longer more likely to classify the novel beans as negative (Pietri, Fazio, \& Shook, 2013a). Recalibration, however, does not promote a positivity bias, or result in participants being more likely classify the new beans as positive. Instead, recalibration encourages a more balanced and accurate classification of the novel beans.

In past research, recalibrating attitude generalization tendencies ultimately modified a very basic process that promoted less negative judgments across a range of domains. Pietri et al. (2013a) found that this intervention produced less negative interpretations of ambiguous situations, reduced reported apprehension at the prospect of taking risks, and encouraged riskier behavior in the laboratory (i.e., more likely to pump a balloon to increase its monetary value, while risking that the balloon will pop and, hence, losing the balloon's accumulated value). 
Recalibrating Valence Weighting and Interracial Interaction

Previous research considered the effects of this recalibration intervention on perceptions of objects and personal decision making, whereas the present research investigated the interpersonal and intergroup consequences. Although cognitions about objects and persons have distinctive elements and activate different areas of the brain (Harris \& Fiske, 2006), generalization of associations is a process basic to both object and social cognition. Moreover, within the domain of intergroup processes, because outgroups are perceived to be relatively homogeneous (Mullen \& Hu, 1989), the strong associative links among members would be expected to particularly facilitate the generalization of attitudes toward an exemplar to other members of the group (Henderson-King \& Nisbett, 1996).

In the current research, we investigated how negative attitude generalization tendencies relate to Whites' anticipated emotions during an online interaction (“chat”) with Black partner. Although online interactions involve anonymity and are generally less anxiety arousing than face-to-face interactions (Amichai-Hamburger \& McKenna, 2006), individuals still feel some anxiety at the prospect of interacting with a member of an outgroup (Finchilescu, 2010). Moreover, virtual intergroup interactions can have similar favorable outcomes for improving intergroup interactions as do in-person interactions (Amichai-Hamburger \& McKenna, 2006; Alvídrez, Piñeiro-Naval, Marcos-Ramos, \& Rojas-Solís, 2013). Virtual interactions also represent a rapidly increasing percentage of people’s social interactions. In 2014, 87\% of American adults used the Internet and 74\% of adults used some sort of social networking site (Pew Research Center, 2014).

In an initial pilot study, we aimed to establish that Whites generalize past negative and positive intergroup experiences when forming expectations about an online interaction with a Black individual. In two subsequent experiments, we examined whether the recalibration 
Recalibrating Valence Weighting and Interracial Interaction

paradigm could then serve as an intervention to encourage less negative anticipated emotions associated with an intergroup chat. In both of these main experiments, prior to learning the race of their chat partner, participants engaged in the BeanFest exercise (Fazio et al., 2004, 2015; Pietri et al., 2013a). Half of the participants received objectively accurate feedback in the BeanFest exercise to reduce the extent to which they weighted negative qualities associated with novel stimuli, while the other half performed the very same tasks but without the benefit of feedback. After participants learned the race of their chat partner we measured participants’ anticipated positive and negative emotions during the chat (Experiments 1 and 2).

We hypothesized participants in the BeanFest condition that promoted the recalibration of tendencies to overgeneralize negative associations would experience less negative emotion in anticipation of an intergroup interaction than participants in the control condition. Furthermore, we predicted that, as a consequence of experiencing less negative emotion, they would be more willing to engage in intergroup contact with a Black person (Plant, 2004; Plant et al., 2008). In Experiment 2, we also investigated whether recalibration would be more successful for individuals who were more prone towards experiencing intergroup anxiety (i.e., for participants who report less closeness with Black individuals). Previous intergroup contact research has found that the beneficial effects (i.e., improved attitudes) of intergroup contact are most pronounced for individuals who have less experience interacting with outgroup members (Hodson, 2011; Pettigrew, \& Tropp, 2006).

\section{Pilot Study}

Before exploring whether the BeanFest recalibration exercise would impact expectations about an intergroup interaction, we attempted to establish that Whites generalize their past negative experiences when forming expectations about a new intergroup interaction. 
Recalibrating Valence Weighting and Interracial Interaction

Additionally, we aimed to examine whether it is Whites’ previous negative (but not positive) negative experiences that significantly predict their reactions to interacting with a Black individual.

\section{Method}

Participants. We recruited 75 White participants from Mturk Amazon’s Mechanical Turk website for a \$1.50 payment. Four participants were excluded from the analyses for incorrectly identifying the race of their chat partner resulting in a final sample of 71 White participants (74\% female).

Procedure. We advertised the study on Amazon Mechanical Turk (Mturk) as being interested in how individuals form friendships in an online chatroom setting. At the beginning of the study, participants were provided with information about their chat partner ostensibly to “help facilitate the interaction.” The chat partner’s gender was programed to always be the same as the participants' gender. Participants also saw the chat partner's race as “Black or African American", home state as "Wisconsin" and hobbies as "Spending time with friends, reading for fun and watching TV. Bike riding.” After learning about the chat partner, participants responded to questions concerning “their feelings about the upcoming interaction.” Following Mallett et al. (2008), we assessed the extent to which participants thought they would feel (1=not at all to 7=very much) negative emotions (annoyed, resentful, nervous, afraid, and angry) and positive emotions (enthusiastic, relaxed, happy, excited, and cheerful). We calculated the means for the negative emotions $(M=1.78, S D=.85, \alpha=.78)$ and positive emotions $(M=3.89, S D=1.39, \alpha=.90)$, with higher scores indicating greater anticipated negative and positive emotions. Negative and positive emotions were negatively correlated, $r(70)=-.27, p=.022$. 
Recalibrating Valence Weighting and Interracial Interaction

We then informed participants that we were interested in how their previous interactions with people in general may influence their chatroom interaction. Participants rated their agreement (1=strongly disagree to 7=strongly agree) with 18 items concerning to their past experiences with other people. Embedded in these questions were two items assessing past negative intergroup interactions ("In the past, I've have sometimes worried I will say the wrong thing when interacting with people who are a different race or ethnicity than me.”; "In the past, I sometimes have found it difficult to talk with people who are a different race or ethnicity than me”) and two items indexing past positive intergroup interactions (“In the past, I've typically had comfortable interactions with people who are a different race or ethnicity than me”; "In the past I've typically gotten along very well with people who are a different race or ethnicity than me”). The two items representing negative and the two items reflecting positive intergroup experiences were significantly correlated, $r(73)=.80, p<.001$ and $r(73)=.86, p<.001$, and we averaged these items to create a score for past negative $(M=3.59, S D=1.56)$ and past positive $(M=5.56, S D=1.22)$ intergroup experiences.

After participants completed the measures of interest, they were informed they would not take part in a chat interaction, were thanked for their participation, and fully debriefed.

\section{Results}

To explore how participants generalized past positive and negative intergroup experiences when forming negative expectations about the chat with a Black partner, we ran a regression analysis predicting negative emotions minus positive emotions from past negative intergroup experiences and past positive intergroup experiences. We found past negative experiences significantly predicted more negative expectations, $b=.50, t(68)=4.02, p<.001$, whereas past positive experiences did not predicted less negative expectations $b=-.24, t(68)=-$ 
Recalibrating Valence Weighting and Interracial Interaction

1.48, $p=.144 .^{2}$ Thus, Whites' previous negative intergroup experiences primarily shape their expectations about a new online interaction with a Black individual.

\section{Experiment 1}

Now that we established that White participants generalize past intergroup experiences, particularly negative ones, when forming expectations about a new intergroup interaction, we aimed to reduce negative expectations by utilizing the BeanFest recalibration exercise. Specifically, in Experiment 1 participants were randomly assigned to the recalibration condition, in which they underwent training to correct the overweighting of negative associations, or to the control condition, in which they received no training. Then, participants were randomly assigned to learn the race of their chat partner would be Black or White, and they completed measures assessing their anticipated emotions during the interaction. After that, the White participants were given the opportunity to enter or avoid the chat interaction. We hypothesized that recalibration training in the BeanFest exercise would decrease negative emotions about the intergroup chat interaction, which would ultimately promote greater willingness to take part in the chat.

\section{Method}

Participants. One hundred and fifty-two White participants were recruited from MTurk for a $\$ 0.50$ payment. Five participants were excluded from the analyses because they pressed the positive bean key during every trial of the test phase, indicating that they were not taking the task seriously. These participants were equally distributed across partner's race $\left(\chi^{2}[1, N=152]=.06\right.$, $p=.805)$ and condition $\left(\chi^{2}[1, N=152]=.21, p=.649\right)$. Seven participants were also excluded from the analyses because they did not correctly identify the race of their chat partner. These participants were also equally distributed between partner's race $\left(\chi^{2}[1, \mathrm{~N}=152]=.53, p=.529\right)$ and 
Recalibrating Valence Weighting and Interracial Interaction condition $\left(\chi^{2}[1, N=152]=.17, p=.685\right)$. Finally one participant was excluded from the main analyses as an outlier, whose response was 3.4 standard deviations over the mean on a main dependent variable. ${ }^{1}$ This left a final sample of 139 participants (56.8\% female).

Our sample size was determined based on past research utilizing the recalibration exercise, which recruited 30-50 participants per condition. Furthermore, research examining anticipated negativity in intergroup contact has relied on 15-30 per cell (see Mallet et al., 2008).

Procedure. The experiment was again advertised on Amazon Mechanical Turk as being interested in learning and friendship formation. Upon entering the experiment, participants were informed they would play a game called BeanFest, which was actually the recalibration exercise (Pietri et al., 2013a), during which they would have to learn which objects (beans) were good or bad in order to gain and avoid losing points. Participants next learned that in the second part of the experiment they would ostensibly take part in a chat interaction.

Participants were then presented with the recalibration exercise, which was closely modeled from Pietri et al. (2013a). For this exercise, participants first received instructions detailing how to play the BeanFest game and were presented with four examples of beans that would increase and decrease points to help facilitate learning.

Because participants did the recalibration exercise online, we shortened it to ensure participants paid attention. Instead of using a 10 x 10 matrix, we employed a 7 x 7 matrix. We used the five beans in each of the four corners of the smaller matrix as the game beans, and assigned two of the corners a +10 value and two of the corners a -10 value (see Figure $2 a$ and $b$ ). Thus, participants could learn simple rules to remember the values of beans (e.g., circle with few speckles is good, oblong with few speckles is bad; to view exact game beans see Figure 2c). 
Recalibrating Valence Weighting and Interracial Interaction

During the initial game phase, participants were presented with all 20 game beans twice during two blocks. Participants began each block with 50 points. If participants reached 100 points during a block, participants were informed that they won the game. Participants then continued the game, starting again with 50 points. If participants hit zero points, they were told that they lost the game, and restarted the game. Regardless of the number of times participants won or lost the game, they saw the 20 game beans twice across two blocks.

During a trial of the game phase, participants saw a bean in the middle of the screen and decided whether or not to select the bean by pressing the " $\mathrm{D}$ ” key (to not select) or the "L" key (to select). If participants selected the bean they were shown the value of the bean and their net loss or gain (always + or -10 points depending on whether the bean was positive or negative) in the lower right corner of the screen and in the lower left corner of the screen participants saw their score, which went up or down depending on the value of the bean. If participants did not select the bean, they saw the value of the bean, the net gain or loss as 0 , and their current score.

In between the first and second block, participants completed a classification training task during which they quickly identified all 20 game beans as helpful or harmful. Participants received corrective information when they incorrectly identified a bean (e.g., "INCORRECT THIS IS A POSITIVE BEAN!!”).

After the game phase, participants completed the test phase in which they saw a bean and had to quickly identify it has helpful or harmful. The test phase consisted of one block with 10 of the game beans and 24 new beans from the matrix that were not shown during the game but closely resembled both positive and negative game beans (see Figure 2d to view all the novel beans shown during the test phase). The experimental manipulation occurred during this phase. After identifying each bean, participants in the recalibration condition received feedback about 
Recalibrating Valence Weighting and Interracial Interaction

their classification of the bean (e.g., "INCORRECT, THIS IS A NEGATIVE (POSITIVE)

BEAN!” or “CORRECT THIS IS A NEGATIVE (POSITIVE) BEAN!”). Feedback for the new beans was based on their proximity to the good and bad game beans in the matrix. Thus, although the new beans were not assigned a value, they were objectively closer to a good or bad game bean region (see Figure 2a and d). By receiving feedback on their classification of the new beans, participants could slowly develop a more balanced weighting of positive versus negative resemblances (Pietri et al. 2013a). The control condition also classified the same beans but received no feedback.

Next, participants were provided with information about their chat partner. Participants saw the chat partner's race as "Black or African American” in the Black chat partner condition or "White or Caucasian" in White chat partner condition and the same additional information outlined in the pilot study.

Participants then completed the same measures employed in the pilot study to assess their negative emotions $(M=1.92, S D=.86, \alpha=.68)$ and positive emotions $(M=3.82, S D=1.44, \alpha=.92)$

Finally, to measure an actual behavior in this experiment, participants were given the option to exit the experiment and not enter the chat:

Before you enter this interaction, it is important to remember this experiment is voluntary and confidential. The individual will not share any information you discuss with him/her. Furthermore, we do not want anyone to feel obligated to enter a situation in which they feel particularly uneasy, or anxious about. So, we are giving you the opportunity to end the experiment now. We will give you the survey code and you will still receive your payment if you choose to quit. The 
Recalibrating Valence Weighting and Interracial Interaction

individual in the chat room will have no way of knowing you decided to end the experiment early.

We then asked participants whether they were willing to enter the chat ("yes” or "no").

(Overall, 58.3\% of participants decided to enter the chat). Regardless of the participants’ decision, the experiment ended, and participants were debriefed.

\section{Results}

Immediate effects of the recalibration exercise on responses to novel stimuli. Based on previous work that has used the recalibration exercise to create more positive orientations to novel stimuli (Pietri et al., 2013a), we first examined whether participants learned to distinguish the "good" and "bad" game beans well, and then tested whether the recalibration and the control conditions differed in their evaluation of novel bean. We found that the proportion of game beans correctly identified as good or bad was high (over $80 \%$ correct) in both the recalibration condition $(M=.84, S D=.19)$ and the control condition $(M=.83, S D=.18)$ and there was no significant difference between the two conditions, $t(137)=.38, p=.71$, Cohen's $d=.06$.

To test whether participants in the recalibration condition responded less negatively to the novel beans (i.e., the beans not seen during the game; Figure 2d) than did participants in the control condition, we compared participants’ average response to the novel beans ( -1 as negative, +1 as positive). Past research has examined participants' response to the novel beans as a way of measuring the recalibration paradigm’s initial effectiveness (Pietri et al., 2013a). As anticipated, participants in the recalibration condition $(M=.04, S D=.24)$ perceived the new non-game beans less negatively than did participants in the control condition, $M=-.11, S D=.28, t(137)=2.85$, $p=.005$, Cohen's $d=.49$. Whereas participants in the control condition generally perceived the new beans negatively compared to the neutral value of $0, t(68)=-2.42, p=.018$, participants in the 
Recalibrating Valence Weighting and Interracial Interaction

recalibration condition perceived them in a more balanced manner compared to $0, t(69)=1.53$, $p=.130$. These findings, using a shortened online paradigm, replicated past laboratory research (Pietri et al., 2013a).

\section{Effects of recalibration on anticipated emotions during the online chat. We next}

explored whether the recalibration exercise influenced participants' expectations about the online chat with a White and Black partner. We performed a mixed model 2 (condition: control vs. recalibration) x 2 (partner: Black vs. White chat partner) x 2 (emotions: positive and negative) analysis of variance (ANOVA), with condition and partner as between subjects and emotions as within subjects variables. There was a significant effect of emotion valence, $F(1,135)=137.29$, $p<.001, \eta_{\mathrm{p}}{ }^{2}=.504$. Overall, participants anticipated feeling more positive emotions $(M=3.82$, $S D=1.44)$ than negative emotions $(M=1.92, S D=.86)$. There were no other main effects and no two-way interactions ( $p s>.270)$. However, as predicted, the three-way interaction between condition, partner, and emotions was significant, $F(1,135)=4.73, p=.031$, partial $\eta_{\mathrm{p}}{ }^{2}=.034$ (see Table 1 for all means and standard deviations).

For anticipated negative emotions, there was a significant condition x partner interaction $F(1,135)=5.46, p=.021, \eta_{\mathrm{p}}{ }^{2}=.039$. When the partner was Black, participants in the recalibration condition predicted they would feel less negative emotions during the interaction than participants in the control condition, $t(74)=2.88, p=.004$, Cohen's $d=.67, \eta_{\mathrm{p}}{ }^{2}=.10$. In contrast, when the partner was White, there was no difference between participants in the recalibration and control conditions, $t(61)=.50, p=.630$, Cohen's $d=.13, \eta_{\mathrm{p}}{ }^{2}=.004$. From an alternative perspective, participants in the control condition tended to anticipate they would feel more negative emotions in an online chat with a Black partner than a White partner, $t(67)=1.63$, $p=.087$, Cohen's $d=.40 ; \eta_{\mathrm{p}}{ }^{2}=.038$ ). Unexpectedly, participants in the recalibration condition 
Recalibrating Valence Weighting and Interracial Interaction

predicted that they would feel somewhat less negative emotions with a Black partner than a White partner (although this difference was not significant) $t(68)=1.68, p=.116$, Cohen’s $d=.41$, $\eta_{\mathrm{p}}{ }^{2}=.040$. By mitigating the negativity bias characterizing the classification of the novel beans, recalibration decreased the anticipated negative emotions that were associated with Black chat partner interaction. However, as a result of effectively decreasing the negative emotions with the Black partner, the recalibration exercise may have resulted in participants actually anticipating less negative emotions with the Black than White partner.

The pattern of results for anticipated positive emotions in the potential chatroom exchange was generally opposite that for negative emotions, but weaker. For positive emotions, the condition x partner interaction was not significant $F(1,135)=2.22, p=.138, \eta_{\mathrm{p}}{ }^{2}=.016$, and the effect of recalibration was not significant when the chat partner was Black, $t(74)=1.46, p=.141$, Cohen's $d=.34, \eta_{\mathrm{p}}{ }^{2}=.028$ (see Table 1 ). Thus, recalibration primarily affected anticipated negative, but not positive emotions in interactions with Black chatroom partner, and did not influence anticipated emotions with a White chatroom partner.

\section{Effects of recalibration on anticipated intergroup anxiety versus intergroup}

hostility. Our index of predicted negative emotions appeared to assess to two components predicted anxiety (i.e., nervous, afraid) and predicted hostility (i.e., angry, resentful, angry) during the intergroup interaction. ${ }^{3}$ Thus, we ran additional exploratory analyses examining the effect of condition and chat partner on predicted anxiety emotions $(M=2.32, S D=1.38, r=.65)$ and predicted hostility emotions ( $M=1.65, S D=.96, \alpha=.79)$. For anxiety emotions, there was a significant effect of condition, $F(1,135)=5.22, p=.024, \eta_{\mathrm{p}}{ }^{2}=.037$, no effect of chat partner, $F(1,135)=1.05, p=.308, \eta_{\mathrm{p}}{ }^{2}=.008$, and no significant interaction between the two $F(1,135)=2.05$, $p=.155, \eta_{\mathrm{p}}{ }^{2}=.015$. However, the pattern of results was in the expected direction. Importantly, 
Recalibrating Valence Weighting and Interracial Interaction

with the Black chat partner there was a strong effect of recalibration $t(74)=2.50, p=.007$, Cohen's $d=.58, \eta_{\mathrm{p}}{ }^{2}=.08$, whereas in the White chat partner condition there was no effect of recalibration, $t(61)=.67, p=.564$, Cohen's $d=.14, \eta_{\mathrm{p}}{ }^{2}=.007$. For hostile emotions there was no effect of condition $F(1,135)=.11, p=.746, \eta_{\mathrm{p}}{ }^{2}=.001$ or chat partner $F(1,135)=.53, p=.468, \eta_{\mathrm{p}}{ }^{2}=.004$, but a significant interaction between the two $F(1,135)=4.13, p=.044, \eta_{\mathrm{p}}{ }^{2}=.030$. However, and interestingly, with the Black chat partner there was only a marginal effect of recalibration condition, $t(74)=1.76, p=.082$, Cohen's $d=.41, \eta_{\mathrm{p}}{ }^{2}=.04$, and as expected, no effect of recalibration with White partner, $t(61)=1.15, p=.250$, Cohen's $d=.29, \eta_{\mathrm{p}}{ }^{2}=.021$. Thus, recalibration significantly decreased intergroup anxiety, and only marginally reduced predicted intergroup hostility. It also worth noting that in the control condition with a Black partner, participants anticipated feeling significantly more anxious emotions than hostile emotions, $t(37)=3.45$, $p=.001$ (see Table 1).

Effects of recalibration on decision to enter the chatroom. A logistic regression analyses testing the effects of condition and partner on whether participants decided to engage in the online chat (Yes or No) showed that, overall, participants in the recalibration condition (66\%) tended to be more likely to enter the chat than participants in the control condition (51\%), $b=.62$, $Z=1.78, p=.076$, but did not reveal the anticipated interaction, $b=.31, Z=.44, p=.66$ (see Table 1).

Although there was no total effect on the decision to enter the chatroom, we examined whether recalibration had an indirect effect on decision to enter to chatroom via participants' anticipated anxious emotions (i.e., the emotions most impacted by recalibration). Supporting this possibility, anxious emotions significantly negatively correlated with decision to enter the chat, $r(138)=-.21, p=.015$. We, thus, ran a moderated mediation analysis using Hayes’s (2013) Process macro model 7 and 10,000 bootstrap resamples (see Figure 3). We found when the chat partner 
Recalibrating Valence Weighting and Interracial Interaction

was Black, for condition there was a significant indirect effect (i.e., the confidence interval did not include 0 ) of anticipated anxious emotions on participants' decision to enter the chat (.12; 95\% CI: .004,.37). That is, when the chat partner was Black, participants in the recalibration condition, compared to those in the control condition, predicted they would feel less anxious during the chat and, in turn, anticipating being less anxious related to a greater likelihood of entering the chatroom. However, when the chat partner was White, the comparable indirect effect was not significant (.02; 95\% CI: -.05,.15).

\section{Discussion}

In Experiment 1, we found the training administrated during recalibration helped participants generalize negative attitudes less strongly when classifying the novel beans, which then promoted less anticipated negative emotions during a chat with a Black individual. Perhaps because the recalibration exercise operated primarily by allaying the negativity bias participants showed when classifying the novel beans but did not promote a positivity bias (i.e., participants did not classify the beans as significantly more positively compared to the 0 neutral point), recalibration was more effective at reducing anticipated negative emotions than increasing anticipated positive emotions.

Recalibration also appeared to be more effective at modifying intergroup anxiety related emotions than intergroup hostility emotions. Traditionally, Whites’ biases toward Blacks have been characterized as reflecting “antipathy,” often including intense feelings of anger and hatred (Allport, 1954). However, contemporary forms of racial bias among Whites toward Blacks often involve feelings of anxiety and discomfort (Dovidio \& Gaertner, 2004; Smith \& Mackie, 2005). Relatedly, in the current experiment, participants in the control condition who were going to interact with a Black chat partner reported more anxious than hostile emotions. Intergroup 
Recalibrating Valence Weighting and Interracial Interaction

anxiety is also characterized by greater ambivalence (i.e., one should have egalitarian values, but one feels discomfort when interacting with Black individuals) than intergroup hostility (i.e., one feels anger and hatred toward Black individuals) (Dovidio \& Gaertner, 2004; Gaertner \& Dovidio, 1986). Thus, these anxious feelings may be the ideal set of emotions to target with recalibration.

\section{Experiment 2}

The primary goal of Experiment 2 was to replicate our finding that recalibration reduced anticipated intergroup anxiety. Because we did not find an effect of recalibration in the samerace chat partner condition in Experiment 1, in Experiment 2 we focused only on White participants’ reactions to interacting with a Black chat partner. We again tested whether, compared to the control condition, recalibration would result in less anticipated intergroup anxiety during a chat.

A secondary aim of Experiment 2 was to examine whether recalibration would be more effective for particular individuals. We specially tested whether the effect of recalibration was moderated by past close relationships with Black individuals. There has been a vast amount of research demonstrating lack of contact with outgroup members predicts higher intergroup anxiety (see Pettigrew \& Tropp, 2011). As a result, positive contact effects are strongest among individuals with less previous intergroup interactions (Hodson, 2011; Pettigrew, \& Tropp, 2006). Thus, individuals who report few close relationships with Blacks, may likely also have more uncomfortable and fewer enjoyable past intergroup encounters. As a result, they may strongly benefit from training to give less weight to negative associations (in this case, uncomfortable previous intergroup experiences) when forming an assessment of new interaction with a Black individual. Furthermore, past research on the weighting of positive and negative associations 
Recalibrating Valence Weighting and Interracial Interaction

found differences in valence weighting was more predictive of the likelihood of engaging in risktaking events (e.g., chasing tornados in order to take dramatic photos) with which participants had had little experience in the past (Pietri et al., 2013b). When people judge a future situation they have frequently encountered in the past, they can rely on their memories when making predictions about this event. Thus, we predicted recalibration may be less influential for Whites who already have close relationships with Blacks because they could use their memories of these relationships to form expectations about the chat.

In Experiment 2 we also increased the number of participants recruited in each condition in order to ensure the robustness of the effects and ask more precise vetting questions. Specifically, because the interaction supposedly would occur via an online chatroom, we reasoned that some participants might not believe they would actually engage in a chat. Thus, recruiting a higher number of participants permitted us to assess whether participants believed they would engage in a real chat, and exclude participants who did not trust our cover story. We ran a power analyses using $\mathrm{G}^{*}$ power and based on the effect size between the recalibration and control condition on anxious emotions $(d=.58)$, and found to reach $85 \%$ power, we would require approximately 110 participants.

\section{Method}

Participants. We recruited 178 White participants from Mturk for \$1.50 payment. Four participants (2\%) were excluded from the analyses because they pressed only one button during all of the BeanFest game and test phases, indicating they were not taking the task seriously. These participants were equally distributed across conditions $\left(\chi^{2}[1, N=178]=.00, p=.973\right)$. An additional nine participants (5\%) were excluded from the analyses because they did not correctly identify the chat partner’s race as Black, and these participants were also equally distributed 
Recalibrating Valence Weighting and Interracial Interaction

across condition $\left(\chi^{2}[1, N=178]=.08, p=.772\right)$. Finally, 41 (23\%) of participants did not believe that they were going to interact with a real chat partner (i.e., answered "no" instead of "yes" or “maybe” on the question “Did you believe you were going to have a real chat conversation?”), and thus were not included in the final analyses. These participants were also equally distributed across the each condition $\left(\chi^{2}[1, N=178]=.25, p=.620\right){ }^{4}$ This left us with a final sample of 124 (61 in the control condition, and 63 in the recalibration), which was above our desired number of participants.

Procedure. Participants first completed the same BeanFest game and recalibration paradigm employed in Experiment 1. Half of the participants received feedback during every trial of the test phase (the recalibration condition), whereas the other half did not (the control condition). Following BeanFest, participants were provided with the same information utilized in Experiment 1 about their chat partner. However, in the current experiment, all participants were told that their chat partner would be Black. Participants then completed the same measures employed in Experiment 1 to assess their anticipated emotions. Based on the results from Experiment 1, we created a measure of anticipated negative emotions, $(\alpha=.75, M=1.71, S D=.91)$, anxious emotions $(M=2.05, S D=1.23, r=.52)$, hostile emotions $(M=1.43, S D=.77, \alpha=.80)$, and positive emotions $(M=4.19, S D=1.55, \alpha=.93)$.

After completing the primary outcome measures, as in Experiment 1, participants were given the option to enter or avoid the chat interaction (57.3\% decided to enter). Participants then answered two items from Tropp and Pettigrew (2005), assessing their level of closeness with Black individuals on a 1= not at close to 7=very close scale ("How close do you feel to the Black people you know?”; “How close do you feel to the Black people you have as friends?”) ( $r=.76$, $M=4.86, S D=1.55)$. Participants completed this measure at the end of the experiment because we 
Recalibrating Valence Weighting and Interracial Interaction

did not want these questions to inadvertently give away the true purpose of the experiment.

However, participants' answers did not vary as a function of recalibration condition $t(122)=.10$, $\mathrm{p}=.918, d=.02$. Finally, participants were thanked for their participation and fully debriefed.

\section{Results}

\section{Immediate effects of recalibration on the weighting of positives and negatives. We} again first examined how well participants learned the "good" and "bad” game beans, and then tested whether the recalibration and the control conditions differed in their classification of novel beans. The proportion of game beans correctly identified was again high (over $80 \%$ correct) across both the recalibration $(M=.83, S D=.17)$ and the control condition $(M=.83, S D=.18)$, and there was no difference between the two conditions, $t(122)=.17, p=.863$, Cohen's $d=.03$. Participants in the recalibration condition $(M=-.03, S D=.26)$ tended to classify the new non-game beans less negatively than did participants in the control condition $(M=-.10, S D=.24$; although this difference did not reach significance) $t(122)=1.55, p=.123$, Cohen’s $d=.28$. Participants in the control condition showed a negativity bias (i.e., compared to 0 ) $t(60)=-3.45, p=.001$, whereas participants in the recalibration condition perceived the novel beans in a more balanced manner, $t(62)=-1.05, p=.300$.

Effects of recalibration on anticipated anxious emotions. Because in Experiment 1 recalibration was most effective at reducing anticipated anxious emotions, for the remainder of the analyses we focused specifically on these emotions. We again found the effects of recalibration were much weaker or non-existent with hostile and positive emotions. ${ }^{5}$

However, replicating Experiment 1, we found that compared to the control condition, participants in the recalibration condition anticipated experiencing significantly less anxious emotions with the Black partner, $t(122)=2.24, p=.027$, Cohen’s $d=.40$. 
Recalibrating Valence Weighting and Interracial Interaction

Effects of recalibration on decision to enter the chatroom. We did not find a direct

effect of recalibration condition on decision to enter the chat, $\chi^{2}[1, N=122]=.57, p=.452$.

However, anxious emotions again negatively correlated with participants' decision to enter the chat, $r(123)=-.25, p=.006$. Thus, we tested the indirect relationship, from the recalibration manipulation through anxious emotions to the decision to enter the chatroom using Hayes's PROCESS macro, model 4, and 10,000 bootstrap resamples (see Figure 4). We found a significant indirect of condition on decision to enter the chat via anticipated anxious emotions (.24; 95\% CI: .03, .59).

Moderation by past close relationships with Blacks. To test the hypothesis that the recalibration effect would be stronger among participants with relatively less close relationships with Blacks, we next ran a linear regression analyses predicting anxious emotions from condition (recalibration=-.5, control=.5), closeness with Black people (mean centered), and the interaction between the two. There was a significant effect of recalibration condition, $b=-.49, t(120)=-2.25$, $p=.026$, and no effect of closeness, $b=.02, t(120)=.21, p=.831$. Although the anticipated two-way interaction was only marginally significance, $b=.24, t(120)=1.75, p=.083$, as suggested by Rosenthal and Rosnow (1985), we conducted tests of our focused predictions separately by examining the effects of recalibration for people who reported relatively less and more close relationships with Black people. For participants with lower reported closeness (one standard deviation below the mean), the recalibration condition (compared to the control condition) produced lower levels of anticipated anxious emotions, $b=-.86, t(120)=-2.83 p=.006$. In contrast, for participants who were more close to Black people (one standard deviation above the mean), there was no effect of recalibration, $b=-.11, t(120)=-.35, p=.727$ (see Figure 5). Thus, 
Recalibrating Valence Weighting and Interracial Interaction

recalibration was more successful at reducing anticipated anxious emotions for participants who reported less closeness with Black individuals.

\section{Discussion}

Replicating Experiment 1, in Experiment 2 we found that compared to the control condition, participants in the recalibration condition were less negative in their classification of novel beans and anticipated experiencing less anxious emotions when interacting with a Black chat partner, which ultimately related to participants' decision to enter the chat. Additionally, recalibration was more successful for participants who reported less close relationships with Blacks.

\section{General Discussion}

Despite the benefits of intergroup contact for improving Whites’ racial attitudes, Whites tend to avoid opportunities for interracial contact because they generally experience anxiety about interracial interactions (Plant, 2004). To address this problem, we utilized a recalibration exercise (Fazio et al., 2015; Pietri et al., 2013b) to modify the extent to which Whites generalize negative attitudes when evaluating new objects. In a pilot study, we demonstrated that Whites on average tended to generalize their past negative (but not positive) intergroup experiences when developing expectations about a new intergroup interaction. Across two subsequent experiments we found that participants who underwent the recalibration exercise, compared to those in a control condition, anticipated less anxious emotions during the interracial chat, which ultimately predicted participants' decision to participate in the chat. In Experiment 2, we found the positive effect of recalibration (i.e., decreased anxiety) were stronger for participants who had fewer close relationships with Blacks, and who likely also had more negative than positive past intergroup experiences. As a result, these participants appeared to receive more benefit from recalibration to 
Recalibrating Valence Weighting and Interracial Interaction

give less weight to negative associations (i.e., uncomfortable previous intergroup experiences) when forming expectations about a new interaction with a Black individual.

Thus, by giving participants intense and repeated trial-by-trial feedback about the extent to which they appropriately weight resemblance to a known positive versus negative, the recalibration exercise provided Whites feedback they rarely receive under naturalistic circumstances (especially if they have little interactions Black individuals), and helped them avoid overgeneralizing negative information when evaluating novel objects and a new intergroup interaction (Fazio et al., 2004; Mallett \& Wilson, 2010; Rocklage \& Fazio, 2014). In the current experiments, we believe recalibrating individuals to generalize positive and negative associations equally when classifying novel beans modified a basic process that promoted generalizing the negative associations with intergroup contact (i.e., past unpleasant intergroup interactions) less heavily, resulting in less negative expectations about a new intergroup experience.

Consistent with the critical role of anxiety in shaping intergroup relations (Dovidio \& Gaertner, 2004; Smith \& Mackie, 2005), we found anticipated anxiety emotion (not positive emotion or hostile emotion) represented the key element in the path from the recalibration manipulation to Whites’ willingness to engage in online contact with a Black person. One reason why anxiety emotion may be a particularly potent factor in the context of this decision is that anxiety, as an emotion, generally, motivates avoidance of stimuli that elicit the emotion (Carver \& Harmon-Jones, 2009). Moreover, participants reported stronger anxious than hostile emotions about chat, making these feelings of intergroup anxiety a good target for recalibration. Additionally, recalibration may have been less effective at promoting positive emotions because the recalibration feedback during test phase of BeanFest mitigated the negativity bias when participants classified the novel beans, but did not encourage a positivity bias. 
Recalibrating Valence Weighting and Interracial Interaction

The current research theoretically offers a broader perspective on the origins of bias and intergroup anxiety. For example, past research found a link between the likelihood of associating negative affect with new objects and prejudice tendencies (Livingston \& Drwecki, 2007), and the current research further reveals that the general overweighting of negative associations may be an underlying reason for avoidance of intergroup contact. Similar to how individuals tend overweight negative information when generalizing their attitudes toward novel objects that bear some resemblance to past good and bad objects, White people on average, tended to overgeneralize past negative intergroup experiences when forming expectations about new interactions with Blacks.

However, it is important to note that individual differences most likely moderate this negativity bias affecting intergroup interactions. In the current research, recalibration was less effective for participants with close relationships with Black people. Additionally, previous research has demonstrated that for individuals who are intrinsically motivated to act in line with their egalitarian values (i.e., self-determined prejudice regulators) threatening information about an outgroup (e.g., that they were taking desired jobs) did not influence their overall level of intergroup anxiety (Legault \& Green-Demers, 2012). As a result, it may be particularly beneficial to target the recalibration paradigm to Whites who overgeneralize negative information (i.e., individuals with few close Black friends, non-self-determined prejudice regulators) when forming expectations about interacting with Blacks.

The current experiments also add to the past research on recalibrating individuals to give equal weight to positive and negative information. Specifically, much of the previous experiments examined recalibration's effect on self-reported feelings towards a hypothetical situation (Pietri et al., 2013a, Experiments 1-3). The present research demonstrated the social 
Recalibrating Valence Weighting and Interracial Interaction

effects of recalibration, specifically in terms of influencing feelings about an interaction with another person (the online chat) and behavior (the decision to enter the chat). Furthermore, this experiment extended recalibration research into the new and important domain of intergroup relations.

We acknowledge that the present research involved only one perspective in potential interracial interaction, the perspective of Whites and only focused on Black-White intergroup relations in the US. However, intergroup interactions also represent a risk for Blacks because they could interact with a White person who is racist and undervalues them (Shelton, Richeson, \& Salvatore, 2005; Shelton, Richeson, \& Vorauer, 2006). Thus, the effect of recalibration may also operate similarly for Black individuals, reciprocally promoting positive intergroup orientations.

Future research might also explore the recalibration procedure's effect on intergroup attitudes more generally. Specifically, the recalibration procedure could potentially promote the formation of positive attitudes toward Black individuals and the transfer of these feelings to Blacks as a group. When Whites enter interracial interactions with more negative expectations, even if the interaction goes well Whites are less likely to generalize this specific positive experience with a Black person to Blacks as a whole (Deegan, Hehman, Gaertner, \& Dovidio, 2015). Thus, using the recalibration paradigm to reduce expectations about intergroup interactions may encourage the formation and generalization of positive attitudes toward Blacks as a group.

In conclusion, the current research utilized the recalibration exercise to reduce Whites’ anticipated intergroup anxiety, and consequent avoidance of an online interaction with Black partner. It may represent a useful element of a multi-stage intervention to improve socially 
Recalibrating Valence Weighting and Interracial Interaction

valued intergroup relations, and as noted earlier, it may be a particular useful intervention when targeted towards certain individuals. In particular, our finding that the recalibration was more beneficial for Whites who had fewer close relationships with Blacks is promising because most Whites in the US have limited experiences with Blacks (e.g., 40\% White individuals report having no friends of different ethnicities; Reuters, 2013). Thus, the recalibration exercise has the potential to be a subtle and valuable intervention in changing expectations about and ultimately promoting intergroup contact. 
Recalibrating Valence Weighting and Interracial Interaction

\section{References}

Allport, G. W. (1954). The nature of prejudice. Reading, MA: Addison Wesley.

Alvídrez, S., Piñeiro-Naval, V., Marcos-Ramos, M., \& Rojas-Solís, J. L. (2014). Intergroup contact in computer-mediated communication: The interplay of a stereotypedisconfirming behavior and a lasting group identity on reducing prejudiced perceptions. Computers in Human Behavior. Retrieved from http://dx.doi.org/10.1016/ j.chb.2014.09.006

Amichai - Hamburger, Y., \& McKenna, K. Y. (2006). The contact hypothesis reconsidered: Interacting via the Internet. Journal of Computer-Mediated Communication, 11(3), 825843. doi: 10.1111/j.1083-6101.2006.00037.x

Apfelbaum, E. P., Sommers, S. R., \& Norton, M. I. (2008). Seeing race and seeming racist? Evaluating strategic colorblindness in social interaction. Journal of Personality and Social Psychology, 95(4), 918-932. doi: 10.1037/a0011990

Carver, C. S., \& Harmon-Jones, E. (2009). Anger is an approach-related affect: evidence and implications. Psychological bulletin, 135(2), 183-204.

Dovidio, J. F., \& Gaertner, S. L. (2004). Aversive racism. In M. P. Zanna (Ed.), Advances in experimental social psychology (Vol. 36, pp. 1-51). San Diego, CA: Academic Press.

Dovidio, J. F., Gaertner, S. L., \& Kawakami, K. (2003). Intergroup contact: The past, present, and the future. Group Processes \& Intergroup Relations, 6(1), 5-21. doi: $10.1177 / 1368430203006001009$

Fazio, R. H., Jackson, J. R., Dunton, B. C., \& Williams, C. J. (1995).Variability in automatic activation as an unobtrusive measure of racial attitudes: A bona fide pipeline? Journal of Personality and Social Psychology, 69, 1013-1027. doi: 10.1037/0022-3514.69.6.1013 
Recalibrating Valence Weighting and Interracial Interaction

Fazio, R. H., Eiser, J. R., \& Shook, N. J. (2004). Attitude formation through exploration: valence asymmetries. Journal of Personality and Social Psychology, 87(3), 293-311. doi: 10.1037/0022-3514.87.3.293

Fazio, R. H., Pietri, E. S., Rocklage, M.R. \& Shook, N. J. (2015). Positive versus negative valence: Asymmetries in attitude formation and generalization as fundamental individual differences. In J. M. Olson \& M. P. Zanna (Eds.), Advances in Experimental Social Psychology (Vol. 51, pp. 97-146). Burlington: Academic Press.

Finchilescu, G. (2010). Intergroup anxiety in interracial interaction: The role of prejudice and metastereotypes. Journal of Social Issues, 66(2), 334-351. doi: 10.1111/j.15404560.2010.01648.x

Harris, L. T., \& Fiske, S. T. (2006). Dehumanizing the lowest of the low: Neuroimaging responses to extreme out-groups. Psychological Science, 17, 847-853. doi: 10.1111/j.1467-9280.2006.01793.x

Henderson-King, E. I., \& Nisbett, R. E. (1996). Anti-black prejudice as a function of exposure to the negative behavior of a single black person. Journal of Personality and Social Psychology, 71(4), 654-664. doi: 10.1037/0022-3514.71.4.654

Lavine, H., Burgess, D., Snyder, M., Transue, J., Sullivan, J. L., Haney, B., \& Wagner, S. H. (1999). Threat, authoritarianism, and voting: An investigation of personality and persuasion. Personality and Social Psychology Bulletin, 25(3), 337-347. doi: $10.1177 / 0146167299025003006$

Legault, L., \& Green-Demers, I. (2012). The protective role of self-determined prejudice regulation in the relationship between intergroup threat and prejudice. Motivation and Emotion, 36(2), 143-158. doi: 10.1007/s11031-011-9242-9 
Recalibrating Valence Weighting and Interracial Interaction

Livingston, R. W., \& Drwecki, B. B. (2007). Why are some individuals not racially biased?

Susceptibility to affective conditioning predicts nonprejudice toward Blacks. Psychological Science, 18(9), 816-823. doi: 10.1111/j.1467-9280.2007.01985.x

Mallett, R. K., \& Wilson, T. D. (2010). Increasing positive intergroup contact. Journal of Experimental Social Psychology, 46(2), 382-387. doi: 10.1016/j.jesp.2009.11.006

Mallett, R. K., Wilson, T. D., \& Gilbert, D. T. (2008). Expect the unexpected: failure to anticipate similarities leads to an intergroup forecasting error. Journal of Personality and Social Psychology, 94(2), 265-277. doi: 10.1037/0022-3514.94.2.94.2.265

Migacheva, K., \& Tropp, L. R. (2012). Learning orientation as a predictor of positive intergroup contact. Group Processes \& Intergroup Relations, 16(4), 426-444. doi:

$10.1177 / 1368430212455854$

Mullen, B., \& Hu, L. (1989). Perceptions of ingroup and outgroup variability: A meta-analytic integration. Basic and Applied Social Psychology, 10, 233-252. doi: 10.1111/j.14679221.2009.00733.x

Paolini, S., Hewstone, M., Cairns, E., \& Voci, A. (2004). Effects of direct and indirect crossgroup friendships on judgments of Catholics and Protestants in Northern Ireland: The mediating role of an anxiety-reduction mechanism. Personality and Social Psychology Bulletin, 30(6), 770-786. doi: 10.1177/0146167203262848

Pettigrew, T. F. (1998). Intergroup contact theory. Annual Review of Psychology, 49(1), 65-85. doi: 10.1146/annurev.psych.49.1.65

Pettigrew, T. F., \& Tropp, L. R. (2006). A meta-analytic test of intergroup contact theory. Journal of personality and social psychology, 90(5), 751-783. doi: 10.1037/00223514.90.5.751 
Recalibrating Valence Weighting and Interracial Interaction

Pettigrew, T. F., \& Tropp, L. R. (2011). When groups meet: The dynamics of intergroup contact. New York: Psychology Press.

Pew Research Center (2014). Internet use over time. [Data file]. Retrieved from http://www.pewinternet.org/data-trend/internet-use/internet-use-over-time/

Pew Research Center (2014). Social media use over time. [Data file]. Retrieved from http://www.pewinternet.org/data-trend/social-media/social-media-use-all-users/

Pietri, E. S., Fazio, R. H., \& Shook, N. J. (2013a). Recalibrating positive and negative weighting tendencies in attitude generalization. Journal of Experimental Social Psychology, 49(6), 1100-1113. doi: 10.1016/j.jesp.2013.08.001

Pietri, E. S., Fazio, R. H., \& Shook, N. J. (2013b). Weighting positive versus negative: The fundamental nature of valence asymmetry. Journal of Personality, 81(2), 196-208. doi: 10.1111/j.1467-6494.2012.00800.x

Plant, E. A. (2004). Responses to interracial interactions over time. Personality and Social Psychology Bulletin, 30(11), 1458-1471. doi: 10.1177/0146167204264244

Plant, E. A., \& Butz, D. A. (2006). The causes and consequences of an avoidance-focus for interracial interactions. Personality and Social Psychology Bulletin, 32(6), 833-846. doi:

Plant, E. A., Butz, D. A., \& Tartakovsky, M. (2008). Interethnic interactions: Expectancies, emotions, and behavioral intentions. Group Processes \& Intergroup Relations, 11(4), 555-574. doi: 10.1177/0146167206287182

Reuters (2013). Close friends of different race or ethnicity. [Data file]. Retrieved from http://polling.reuters.com/\#!response/D1B_2/type/smallest/filters/RACE_:1/dates/201307 $\underline{24-20131217}$ 
Recalibrating Valence Weighting and Interracial Interaction

Richeson, J. A., \& Shelton, J. N. (2003). When prejudice does not pay effects of interracial contact on executive function. Psychological Science, 14(3), 287-290. doi: 10.1111/14679280.03437

Rocklage, M. D., \& Fazio, R. H. (2014). Individual differences in valence weighting: When, how, and why they matter. Journal of Experimental Social Psychology, 50, 144-157. doi: 10.1016/j.jesp.2013.09.013

Rosenthal, R, \& Rosnow, R. L., (1985). Contrast analysis: Focused comparisons in the analysis of variance. Cambridge, UK: Cambridge University Press.

Rugh, J. S., \& Massey, D. S. (2014). Segregation in post-civil rights America. Du Bois Review: Social Science Research on Race, 11(02), 205-232.

Shelton, J. N. (2003). Interpersonal concerns in social encounters between majority and minority group members. Group Processes \& Intergroup Relations, 6, 171-185. doi: $10.1177 / 1368430203006002003$

Shelton, J. N., \& Richeson, J. A. (2005). Intergroup contact and pluralistic ignorance. Journal of Personality and Social Psychology, 88(1), 91-107. doi: 10.1037/0022-3514.88.1.91

Shelton, J. N., Richeson, J. A., \& Salvatore, J. (2005). Expecting to be the target of prejudice. Implications for interethnic interactions. Personality and Social Psychology Bulletin, 31, 1189-1202. doi: 10.1177/0146167205274894

Shelton, J. N., Richeson, J. A., \& Vorauer, J. D. (2006). Threatened identities and interethnic interactions. European Review of Social Psychology, 17, 321-358. doi: $10.1080 / 10463280601095240$ 
Recalibrating Valence Weighting and Interracial Interaction

Shook, N. J., \& Fazio, R. H. (2008). Interracial roommate relationships: An experimental field test of the contact hypothesis. Psychological Science, 19, 717-723. doi: 10.1111/j.14679280.2008.02147.x

Shook, N. J., Fazio, R. H., \& Eiser, J. R. (2007). Attitude generalization: Similarity, valence, and extremity. Journal of Experimental Social Psychology, 43(4), 641-647. doi: 10.1016/j.jesp.2006.06.005

Smith, E. R., \& Mackie, D. M. (2005). Aggression, hatred, and other emotions. In J. F. Dovidio, P. Glick, \& L. A. Rudman, On the nature of prejudice: Fifty years after Allport (pp. 377392). Malden, MA: Blackwell Publishing.

Towles-Schwen, T., \& Fazio, R. H. (2003). Choosing social situations: The relation between automatically-activated racial attitudes and anticipated comfort interacting with African Americans. Personality and Social Psychology Bulletin, 29, 170-182. doi: $10.1177 / 0146167202239042$

Tropp, L. R., \& Pettigrew, T. F. (2005). Differential relationships between intergroup contact and affective and cognitive dimensions of prejudice.Personality and Social Psychology Bulletin, 31(8), 1145-1158. doi: 10.1177/0146167205274854

Turner, R. N., Hewstone, M., Voci, A., Paolini, S., \& Christ, O. (2007). Reducing prejudice via direct and extended cross-group friendship. European review of social psychology, 18(1), 212-255. doi: 10.1080/10463280701680297 
Recalibrating Valence Weighting and Interracial Interaction

\section{Footnotes}

${ }^{1}$ Exploratory factor analyses indicated that negative emotions had two components (eigenvalue = 1.95), an anxious emotion factor (with nervous and afraid loading primarily on this factor; loadings > .618) and a hostile emotion factor (with resentful, angry, and annoyed loading primarily on this factor; loadings $>.775$ ). Regression analyses revealed that past negative experiences significantly predicted anxious emotions, $b=.21, t(68)=2.37, p=.021$, but only marginally predicted hostile emotions $b=.10, t(68)=1.77, p=.081$.

${ }^{2}$ When we include the outlier in our analyses we still see an interaction between condition and chat partner race, $F(1,136)=3.89, p=.041, \eta_{\mathrm{p}}{ }^{2}=.028$, predicting negative emotions, however, the effect is weaker.

${ }^{3} \mathrm{~A}$ factor analyses again indicated negative emotions had two components (eigenvalue $=1.68$ ), with an anxious emotion factor (loadings $>.881$ ) and hostile emotion factor (loadings $>.832$ ).

${ }^{4}$ When we add these participants back into the analyses, the recalibration still results in significantly less anxious emotions than the control condition, $t(177)=-2.29, p=.023$, Cohen's $d=.34$.

${ }^{5}$ Compared to the control condition, recalibration resulted in marginally less negative emotions, $t(122)=1.95, p=.053$, Cohen's $d=.35$. However, when looking at just hostility emotions, the recalibration condition reported less anticipated hostility than the control condition, but this difference was non-significant, $t(122)=-.86, p=.394$, Cohen’s $d=.16$. There was also no significant effect of recalibration on positive emotions, $t(122)=-1.46, p=.148$, Cohen's $d=.26$, and the effect was going in opposite of the expected direction (see also Table 1). These findings strongly suggest recalibration is primarily effective at reducing intergroup anxiety. 
Recalibrating Valence Weighting and Interracial Interaction

Table 1. Means and standard deviations across conditions and the two experiments

\begin{tabular}{|c|c|c|c|c|}
\hline \multicolumn{5}{|c|}{ Experiment 1} \\
\hline & \multicolumn{2}{|c|}{ Recalibration } & \multicolumn{2}{|c|}{ Control } \\
\hline & $\frac{\text { Black partner }}{\underline{\mathrm{M}(\mathrm{SD})}}$ & $\frac{\text { White partner }}{\underline{\mathrm{M}(\mathrm{SD})}}$ & $\frac{\text { Black partner }}{\underline{\mathrm{M}(\mathrm{SD})}}$ & $\frac{\text { White partner }}{\underline{\mathrm{M}(\mathrm{SD})}}$ \\
\hline$\frac{\text { Negative }}{\text { Emotions }}$ & $1.65(.75)$ & $1.96(.84)$ & $2.22(.96)$ & $1.86(.80)$ \\
\hline Anxiety Emotions & $2.00(1.34)$ & $2.09(1.18)$ & $2.86(1.63)$ & $2.29(1.13)$ \\
\hline Hostile Emotions & $1.41(.72)$ & $1.85(1.02)$ & $1.79(1.11)$ & $1.58(.85)$ \\
\hline$\frac{\text { Positive }}{\text { Emotions }}$ & $4.09(1.40)$ & 3.66 (1.59) & 3.59 (1.51) & $3.90(1.21)$ \\
\hline$\frac{\text { Decision to enter }}{\text { the chat }}$ & $65.8 \%$ & $65.6 \%$ & $47.4 \%$ & $54.8 \%$ \\
\hline \multicolumn{5}{|c|}{ Experiment 2} \\
\hline & & $\begin{array}{c}\text { Recalibration } \\
\text { M(SD) }\end{array}$ & \multicolumn{2}{|c|}{$\begin{array}{l}\text { Control } \\
\mathrm{M}(\mathrm{SD})\end{array}$} \\
\hline Negative emotions & & $1.55(.65)$ & \multicolumn{2}{|c|}{$1.87(1.11)$} \\
\hline Anxiety Emotions & & $1.80(1.81)$ & \multicolumn{2}{|c|}{$2.30(1.30)$} \\
\hline Hostile Emotions & & $1.38(.63)$ & \multicolumn{2}{|c|}{1.49 (.89) } \\
\hline$\underline{\text { Positive emotions }}$ & & $4.00(1.74)$ & \multicolumn{2}{|c|}{4.39 (1.31) } \\
\hline Decision to enter the & hat & $54.0 \%$ & \multicolumn{2}{|c|}{$60.7 \%$} \\
\hline
\end{tabular}


Recalibrating Valence Weighting and Interracial Interaction

Positive Game Bean

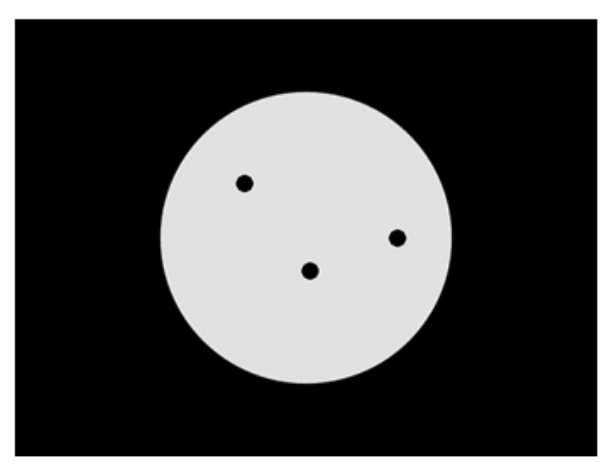

Negative Game Bean

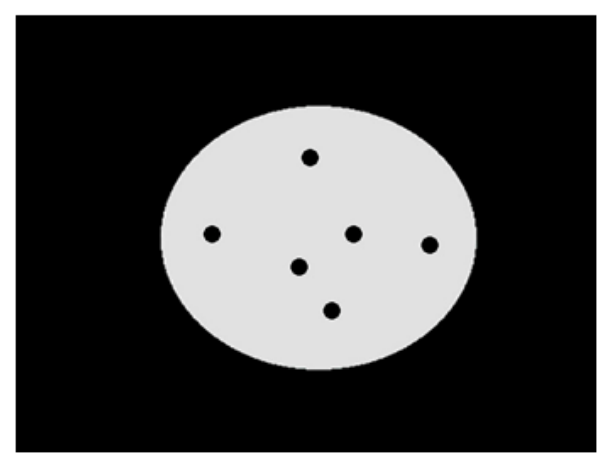

New Bean

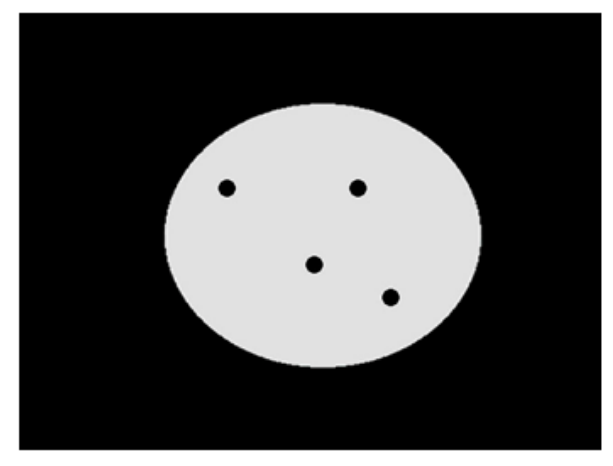

Figure 1. Example of beans shown during the recalibration exercise. On average, without feedback, individuals would classify the new bean as negative. In contrast, because that bean actually more closely resembles the positive game bean (e.g., has four speckles, which is closer to the three speckles on the positive bean than the six speckles on the negative bean) the recalibration exercise would train individuals to classify that bean as positive. 
Recalibrating Valence Weighting and Interracial Interaction

$\mathbf{A}$

\begin{tabular}{|l|l|l|l|l|l|l|l|}
\hline & Y1 & Y2 & Y3 & Y4 & Y5 & Y6 & Y7 \\
\hline $\mathrm{X} 1$ & 10 & 10 & 10 & + & - & -10 & -10 \\
\hline $\mathrm{X} 2$ & 10 & 10 & + & + & - & -10 & -10 \\
\hline $\mathrm{X} 3$ & + & + & + & & - & - & -10 \\
\hline $\mathrm{X} 4$ & - & - & & & & - & - \\
\hline $\mathrm{X} 5$ & -10 & - & - & & + & + & + \\
\hline $\mathrm{X} 6$ & -10 & -10 & - & + & + & 10 & 10 \\
\hline $\mathrm{X} 7$ & -10 & -10 & - & + & 10 & 10 & 10 \\
\hline
\end{tabular}

C

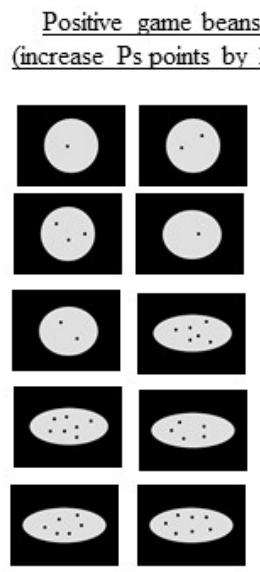

B

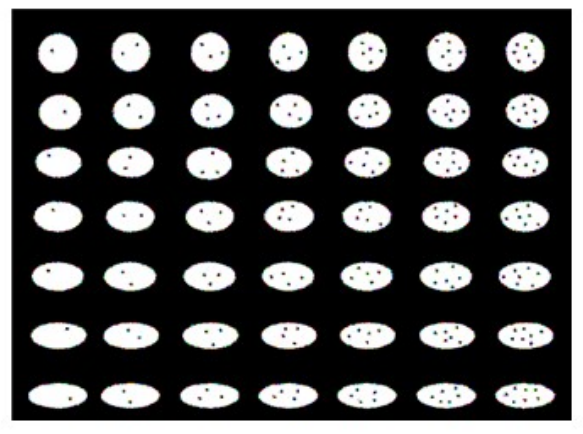

D

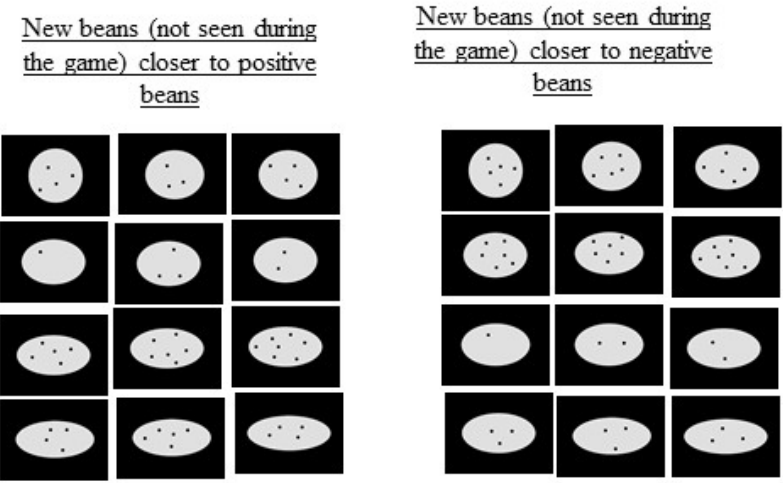

Figure 2. For Figure 2a, X refers to shape from circular to oblong and Y refers to number speckles from one to seven. A -10 value indicates a beans that would decrease participants' points during the game, and a 10 value indicates a beans that would increase participants' points during the game. The spaces with marked by a "-” were novel beans shown during the test phase that were closer to negative game beans, and thus should have been classified as negative. The spaces marked by “+” were also novel beans shown during the test phase, but these beans were closer to positive game beans and should have been classified as positive. The unmarked spaces represent the four beans not presented during the game or test phase. These beans were equal distance from positive and negative game beans, and had no correct classification. Figure $2 b$ through 2d provide visual depictions of beans seen during the game and the test phase. 
Recalibrating Valence Weighting and Interracial Interaction

A

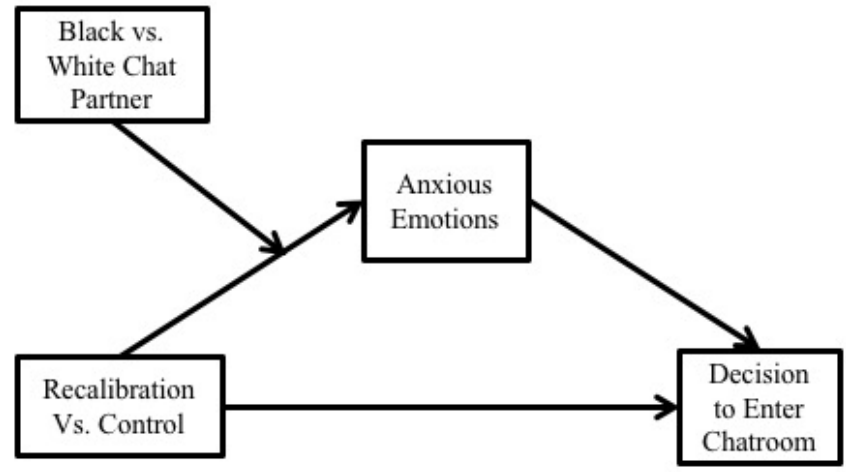

B

Indirect effect $=.12 ; 95 \% \mathrm{CI}: .004, .37$

Black Partner

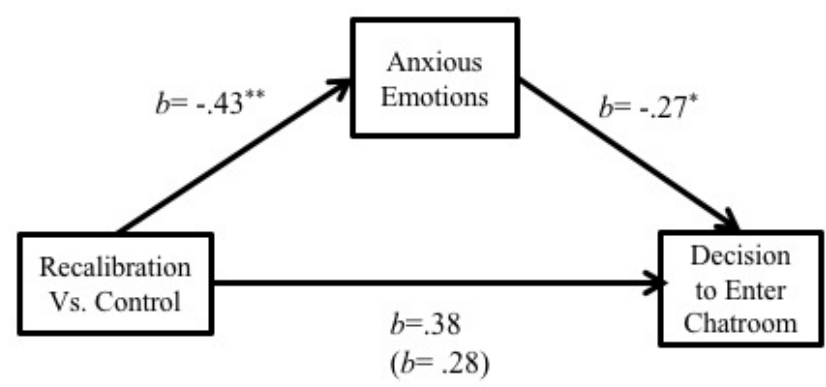

C

White Partner

Indirect effect $=-.02 ; 95 \% \mathrm{CI}:-.05, .15$

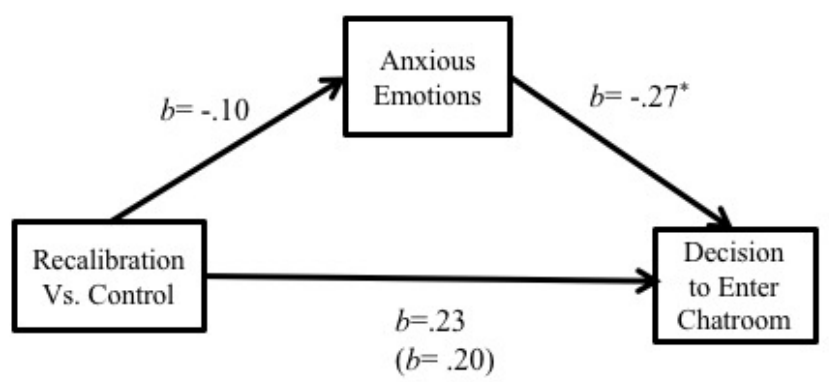

Figure 3. The moderated mediation model testing whether there is an indirect effect of recalibration condition on decision to enter via anticipated anxious emotions with the Black or White partner. Figure 3a demonstrates the conceptual model. Figure 3b shows the mediational model with the Black chat partner, and Figure $3 c$ presents the mediational model with the White chat partner. The total effects are shown without parenthesis, and the direct effects (i.e., controlling for anxious emotions) are shown with parenthesis. $b$ indicates the unstandardized regression coefficient. ${ }^{*}=p<.05,{ }^{* * *}=p<.01$. 
Indirect effect $=.24 ; 95 \% \mathrm{CI}: .03, .59$

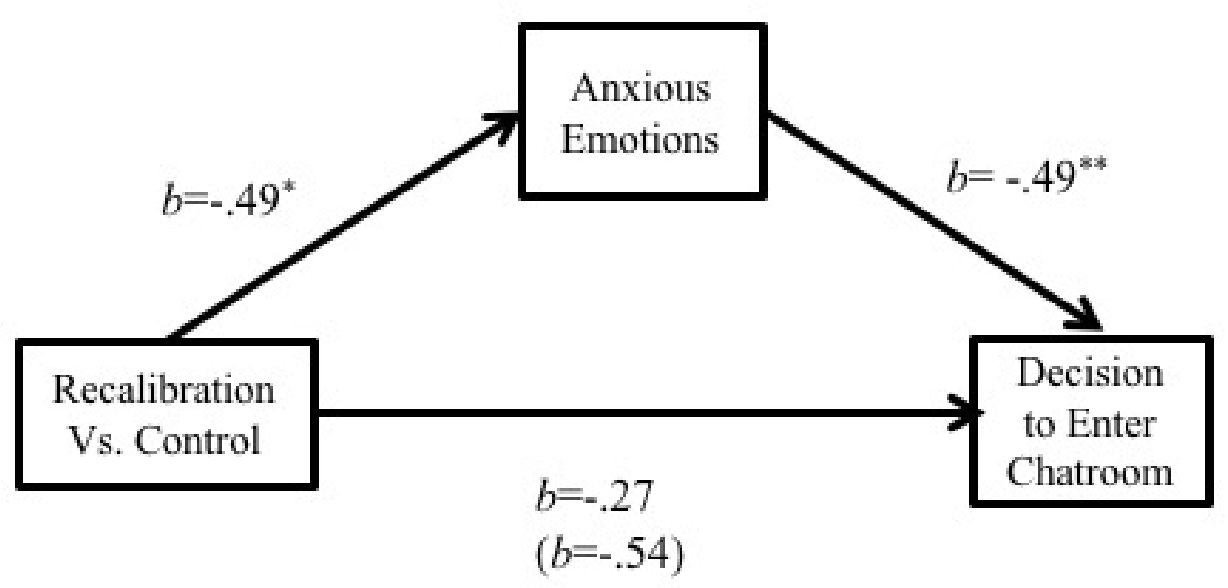

Figure 4. The mediation model testing whether there is an indirect effect of recalibration condition on decision to enter via anticipated anxious emotions. The total effects are shown without parenthesis, and the direct effects (i.e., controlling for negative emotions) are shown with parenthesis. $b$ indicates the unstandardized regression coefficient. ${ }^{*}=p<.05,{ }^{* *}=p<.01$ 


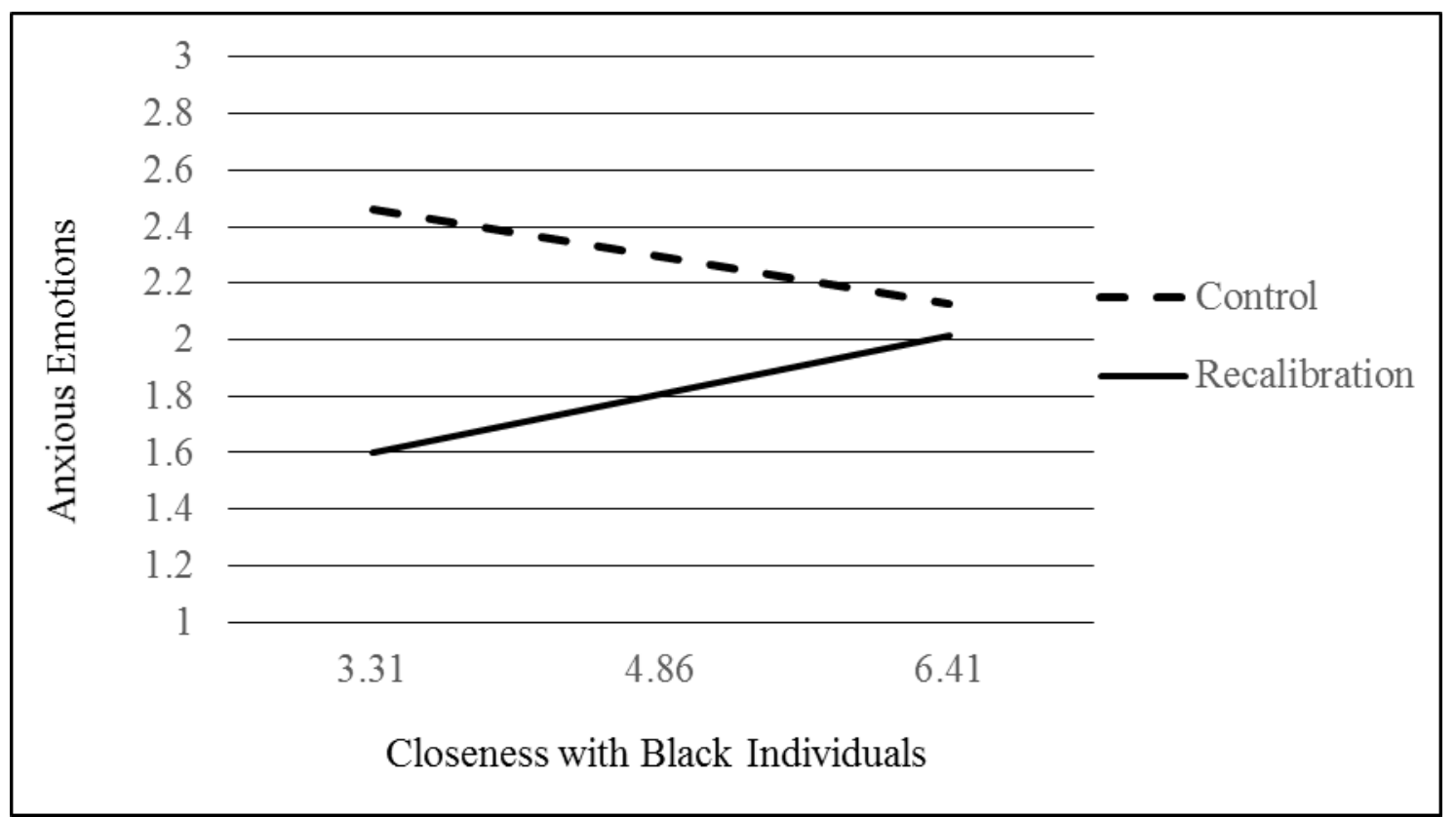

Figure 5. The conditional effect of condition on the probability of entering the chat as a function of closeness with Black individuals. X-axis represents closeness one standard deviation below the mean, at the mean, and one standard deviation above the mean. 